\title{
Impact of electronic conditioning on the noise performance of a two-port network Giant MagnetoImpedance magnetometer
}

\author{
B. Dufay* ${ }^{*}$, S. Saez*, C. Dolabdjian*, A. Yelon ${ }^{\dagger}$, D. Ménard ${ }^{\dagger}$ \\ ${ }^{*}$ GREYC UMR 6072 and Université de Caen Basse-Normandie, France, 14050 Caen Cedex \\ †École Polytechnique de Montréal, département de génie physique \& regroupement québécois des matériaux de \\ pointe, Montréal, Québec, Canada H3C3A7
}

\begin{abstract}
The performance of GMI-based magnetometers is currently limited by the noise due to the electronic conditioning circuitry. We propose a simple model of this noise for a GMI sensor using a synchronous detection scheme. The GMI sensing element consists of a thin pick-up coil wound around a Co-rich amorphous micro-wire. It is fully described by a two port network model and associated impedance matrix. Noise and sensitivity behavior are studied for the four measuring configurations, corresponding to four terms of the impedance matrix. The model yields a good description of experimental data from noise measurements. The magnetic noise spectral density is dominated either by the excitation or detection stages, depending upon whether the excitation currents are high or low. The non-trivial noise behavior exhibited by each configuration leads to better understanding of the noise limitations of GMI magnetometers. The configuration in which the signal at the coil terminals is measured (often named off-diagonal) is the most efficient in decreasing the equivalent output magnetic noise spectral density.
\end{abstract}

Index Terms-Magnetometer, GMI, Noise.

\section{INTRODUCTION}

The Giant Magneto-Impedance (GMI) effect reflects the strong variation of the impedance of a ferromagnetic conductor, driven by a high frequency current, as a function of an external applied magnetic field parallel to the wire. This strong effect, which is very promising for the development of highly sensitive magnetometers [1], [2], [3], [4], [5], has thus attracted considerable attention over the past few years. A very important characteristic of magnetometers is their equivalent magnetic noise level, which ultimately limits the sensing performance. We previously reported that the noise level of current GMI-based magnetometers, in a typical measurement setup, is dominated by the noise of the electronic conditioning, rather than by the intrinsic noise of the GMI sensing element [6], [7], [8]. As a consequence, it is crucial to increase the intrinsic sensitivity of the sensor $\frac{\partial Z}{\partial B}$ (i.e. the differential variation of the impedance expressed in $\Omega / T$ ) until the sensor noise becomes higher than the electronic conditioning noise, in order to further reduce the total equivalent magnetic noise. With that goal in mind, we investigated the noise of a complete GMI device (coupled with thin pick-up coil, or not). Our approach is based on previous work, in which we showed that the GMI-coil set-up, generally labeled off-diagonal GMI [9], [10], orthogonal flux-gate in the fundamental mode [11], [12], or inverse Wiedemann effect [13], is a promising approach to
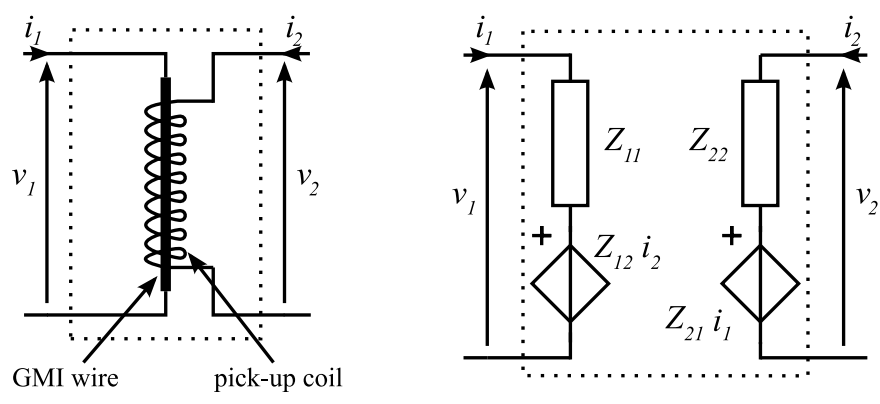

Figure 1. Schematic of the device and associated electrical two port network model. It shows the different terms related to the impedance matrix given in (1).

increasing the intrinsic sensitivity [14]. Here, more attention is dedicated to the noise aspect of the detection configuration, taking into account the electronic conditioning limitation, evaluating the impact of using or not using the coupled pick-up coil. The focus of this article is thus to present the expected noise behavior of a GMI device, including the effect of electronic conditioning. Actual optimization of a complete device, based on the result of this study, will be presented elsewhere.

The paper is organized as follows. Section II describes the electronic setup. The associated noise model is developped in Sec. III. Preliminary results and discussion are presented in section IV. Methods of noise optimisation are discussed in section $\mathrm{V}$, which is followed by a general conclusion.

\section{EXPERIMENTAL METHOD}

Here we present the electronic setup used in our study. We first describe the sensor itself, which consists of a GMI micro-wire coupled to a thin pick-up coil. We then present the electronic conditioning circuit which sets the sensor in appropriate operating conditions.

The sensing element consists of a very thin bi-layer pick-up coil, with approximately 500 turns per layer, directly wound directly around a $100 \mu \mathrm{m}$ diameter $\mathrm{CoFeSiB}$ amorphous ferromagnetic wire. The length of the complete device is approximately $2.5 \mathrm{~cm}$. A schematic of the device along with its equivalent two-port network model are shown in Fig. 1.

The scattering parameters of the device were measured using a classical test setup based on a vector network anal- 
yser [14], driven with sufficiently low excitation power as to insure that the GMI element operated in the linear regime. A description in terms of a field-dependent impedance matrix $[Z(B)]$, where $B\left(B=\mu_{0} H\right)$ is the external magnetic induction, links the voltages to the currents (see Fig. 1 for definitions), that is

$$
\begin{aligned}
\left(\begin{array}{l}
v_{1} \\
v_{2}
\end{array}\right) & =[Z(B)]\left(\begin{array}{l}
i_{1} \\
i_{2}
\end{array}\right) \\
& =\left[\begin{array}{ll}
Z_{11}(B) & Z_{12}(B) \\
Z_{21}(B) & Z_{22}(B)
\end{array}\right]\left(\begin{array}{c}
i_{1} \\
i_{2}
\end{array}\right) .
\end{aligned}
$$

Typical results of measurements of the components of this impedance matrix are illustrated in Fig. 2. Several dc bias currents are superposed on the excitation current, of frequency, $f_{0}=300 \mathrm{kHz}$. This relatively low driving frequency has been chosen in order to minimize coupling (crosstalk) due to wave propagation, despite the improvement in performance expected at higher frequencies. Our focus here is on comprehension of the noise behavior rather than on the optimization of the response. Let us consider that the circuit is operated in a fieldlocked loop configuration. The external magnetic field $B$ is written as

$$
B=B_{0}+b(t)
$$

where $B_{0}$ is the static working point and $b(t)$ is the small magnetic field signal [15]. A first order Taylor expansion allows us to write each term of the impedance matrix as

$$
Z_{i j}(B)=Z_{i j_{0}}+\left.\frac{\partial Z_{i j}(B)}{\partial B}\right|_{B=B_{0}} . b(t)
$$

where $Z_{i j_{0}}=Z_{i j}\left(B_{0}\right)$ and $\left.\frac{\partial Z_{i j}(B)}{\partial B}\right|_{B=B_{0}}$ is the differential impedance variation as a function of the applied magnetic field, expressed in units of $\Omega / \mathrm{T}$. As suggested by the curves of Fig. 2, a dc bias current of $+6 \mathrm{~mA}$ is chosen for the following discussion, in order to enhance the differential impedance variation [6].

In addition to the sensing element, the electronic conditioning circuit consists of an excitation stage, providing an ac current to the two-port network, and a detection stage, which collects the output signal. The schematic of this circuit is shown in Fig. 3. The excitation stage consists of a $300 \mathrm{kHz}$ sine wave voltage generator (HP 33120A), having an amplitude, $e_{g_{1}}$, of $1 \mathrm{~V}_{\mathrm{p}}$. This constant voltage source acts as a constant current source, due to the resistor $R_{1}$. This equivalent sine wave current, which flows through the twoport network, induces a sine wave voltage at output ports. This voltage is modulated in amplitude, with a carrier frequency $f_{0}$, where the modulation depth is directly related to the terms $\left.\frac{\partial Z_{i j}}{\partial B}\right|_{B=B_{0}} . b(t)$ of the impedance matrix.

The detection stage consists of a preamplifier (EG\&G Model 5113) which picks up the voltage at the two-port network terminals. Its use is motivated by the need for high input impedance compared to each $Z_{i j_{0}}$. A large gain, $G \approx 1000$, is chosen, allowing us to neglect any noise sources after the preamplifier. While the proposed model is linear, the experimental behavior for very small $R_{1}$ may not be. To

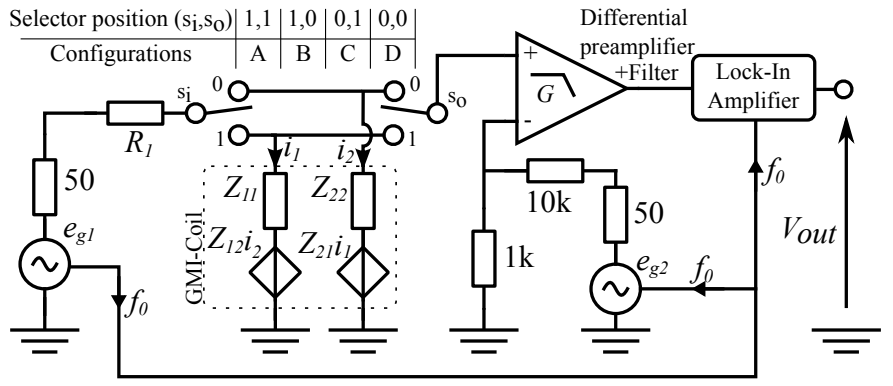

Figure 3. Schematic of the electronic conditioning circuit. The excitation stage uses a voltage generator, $e_{g_{1}}$, and a resistor, $R_{1}$. The detection stage consists of a preamplifier and associated carrier compensation circuitry, followed by a lock-in amplifier locked to the excitation frequency, $f_{0}$.

minimize this problem, a second order low-pass filter with a cut-off frequency of $f_{0}$, follows the preamplifier, in order to suppress any small distortion appearing at high excitation current amplitudes due to non-linear effects in the GMI wire. In order to avoid saturation of the preamplifier and because of this large gain $G$, we need to cancel out the constant average amplitude of the carrier, keeping only the modulated part of the amplitude. This explains the second generator voltage connected to the inverting input of the preamplifier, synchronized to the first, and at the same frequency, with a voltage amplitude, $e_{g_{2}}$.

Following the preamplifier, we use a Stanford Research System (SR 844) Lock-In Amplifier (LIA), locked to the carrier frequency, $f_{0}$. It provides an output signal, directly proportional to the modulation amplitude of the input signal, with gain, $G_{L I A}$. From this description of the system, we have developed a noise model, presented in the next section.

\section{Modeling OF NOISE SOURCES AND EVALUATION OF THE SENSITIVITY}

The noise model developed here allows one to predict noise performance of the setup described in the previous section. First, we quantify the contribution of each noise source, relative to the white noise level at the output of the setup after demodulation. We focus on the voltage spectral density (white noise) in a range around \pm 1 to $10 \mathrm{kHz}$ from the carrier, $f_{0}$, which will be shifted to low frequency after demodulation. Then we predict the output sensitivity, $T_{r}$, in V/T. This links the output signal, $V_{\text {out }}(t)$, to the applied external magnetic field, $b(t)$.

In all of the following, we identify the different excitation/detection configurations by a subscript $X(X=A, B$, $C$, or $D$ ), as defined in Fig. 3 and referring to the impedance matrix term given in Fig. 1. In brief, $A$ means excitation through the amorphous wire with signal detection across its terminals, $B$ means the same excitation, but with detection across the coil terminals. $C$ means the opposite of $B$. $D$ means excitation through the coil combined with detection across its terminals.

Assuming that the intrinsic noise level of the sensing element is lower than that of the conditioning electronic setup [16], we identify three kinds of noise sources. The first is noise induced by the voltage generators, $e_{g}$. Signal instability 



Figure 2. Real and imaginary parts and modulus of $Z_{i j}$ terms of the impedance matrix as a function of the applied magnetic field for three dc bias currents flowing through the amorphous wire. Measurements were performed for an excitation frequency, $f_{0}$, of $300 \mathrm{kHz}$. On the modulus curves, we show estimated differential variations of impedance expressed in $\Omega / \mathrm{T}$. These are used in section III to estimate the sensitivity.

of sinusoidal sources is generally characterized by the singlesideband noise spectral density (NSD) expressed in decibel below the carrier per hertz $\left(\mathrm{dB}_{\mathrm{c}} / \mathrm{Hz}\right)$ [17], in direct relation to the output amplitude of the source. In our case, we consider a dynamic range of $148 \mathrm{dBc} / \mathrm{Hz}$ [18], yielding the voltage power NSD of the generator, expressed as

$$
e_{n g_{1,2}}^{2}=\frac{\left(\frac{e_{g_{1,2}}}{\sqrt{2}}\right)^{2}}{10^{148 / 10}}=\left(\frac{e_{g_{1,2}}}{10^{151 / 20}}\right)^{2}\left[\mathrm{~V}_{\mathrm{rms}}^{2} / \mathrm{Hz}\right]
$$

where $e_{g_{1,2}}$ is the amplitude of generator 1 or 2 in $\mathrm{V}_{\text {peak }}$. The amplitude, $e_{g_{2}}$, of the second generator is related to $e_{g_{1}}$ since amplitude of the signals at both inverting and non-inverting inputs of the preamplifier need to be approximately equal. Consequently, the noise level of the second generator, $e_{n g_{2}}$, may be expressed as functions of $e_{n g_{1}}$ and of circuit elements.

The second noise source to be taken into account is the noise level of the preamplifier, which may be summarized by its $\left(e_{n}, i_{n}\right)$ model, considering an input voltage white power $\mathrm{NSD}, e_{\text {npreamp }}^{2}$, of $\left(3.2 \mathrm{nV}_{\mathrm{rms}} / \sqrt{\mathrm{Hz}}\right)^{2}$ and an input current $\mathrm{NSD}, i_{\text {npreamp }}^{2}$, lower than $\left(20 \mathrm{fA}_{\mathrm{rms}} / \sqrt{\mathrm{Hz}}\right)^{2}$ [19].

The final noise source is related to the Johnson noise of each resistor, $R$, of the setup, expressed as

$$
e_{n R}^{2}=4 k_{B} T R\left[\mathrm{~V}_{\mathrm{rms}}^{2} / \mathrm{Hz}\right]
$$

where $k_{B} \approx 1.38 \times 10^{-23} \mathrm{JK}^{-1}$ is the Boltzmann constant and $T \approx 300 \mathrm{~K}$ is the operating temperature. Assuming that the input impedance of the preamplifier is sufficiently high that we may neglect input current and that the various noise sources are uncorrelated, the white NSD close to the carrier frequency ( $\Delta f \approx \pm 1$ to $10 \mathrm{kHz}$ ) at the LIA input can be obtained from Eqs. (6a) to (6d), for each configuration, $X$ :

In these expressions, noise due to internal generator resistors $(50 \Omega)$ is neglected compared to the generator noise level itself. 


$$
\begin{aligned}
& e_{n L I A_{A}}^{2}\left(f_{0} \pm \Delta f\right) \approx G^{2}\left\{\frac{\left|Z_{11_{0}}\left(f_{0}\right)\right|^{2}}{\left(R_{1}+50+\left|Z_{11_{0}}\left(f_{0}\right)\right|\right)^{2}}\left[2 e_{n g_{1}}^{2}+e_{n R_{1}}^{2}+\left(R_{1}+50\right)^{2} i_{\text {npreamp }}^{2}\right]\right. \\
& \left.+e_{n \text { reamp }}^{2}+e_{n R=1000}^{2}+1000^{2} i_{n \text { preamp }}^{2}\right\} \\
& e_{n L I A_{B}}^{2}\left(f_{0} \pm \Delta f\right) \approx G^{2}\left\{\frac{\left|Z_{21_{0}}\left(f_{0}\right)\right|^{2}}{\left(R_{1}+50+\left|Z_{11_{0}}\left(f_{0}\right)\right|\right)^{2}}\left[2 e_{n g_{1}}^{2}+e_{n R_{1}}^{2}\right]+e_{n R=1000}^{2}\right. \\
& \left.+\left(\left|Z_{22_{0}}\left(f_{0}\right)\right|-\frac{\left|Z_{21_{0}}\left(f_{0}\right)\right|\left|Z_{12_{0}}\left(f_{0}\right)\right|}{R_{1}+50+\left|Z_{11_{0}}\left(f_{0}\right)\right|}\right)^{2} i_{\text {npreamp }}^{2}+e_{\text {npreamp }}^{2}+1000^{2} i_{\text {npreamp }}^{2}\right\}, \\
& e_{n L I A_{C}}^{2}\left(f_{0} \pm \Delta f\right) \approx G^{2}\left\{\frac{\left|Z_{12_{0}}\left(f_{0}\right)\right|^{2}}{\left(R_{1}+50+\left|Z_{22_{0}}\left(f_{0}\right)\right|\right)^{2}}\left[2 e_{n g_{1}}^{2}+e_{n R_{1}}^{2}\right]+e_{n R=1000}^{2}\right. \\
& \left.+\left(\left|Z_{11_{0}}\left(f_{0}\right)\right|-\frac{\left|Z_{21_{0}}\left(f_{0}\right)\right|\left|Z_{12_{0}}\left(f_{0}\right)\right|}{R_{1}+50+\left|Z_{22_{0}}\left(f_{0}\right)\right|}\right)^{2} i_{\text {npreamp }}^{2}+e_{\text {npreamp }}^{2}+1000^{2} i_{\text {npreamp }}^{2}\right\}, \\
& e_{n L I A_{D}}^{2}\left(f_{0} \pm \Delta f\right) \approx G^{2}\left\{\frac{\left|Z_{22_{0}}\left(f_{0}\right)\right|^{2}}{\left(R_{1}+50+\left|Z_{22_{0}}\left(f_{0}\right)\right|\right)^{2}}\left[2 e_{n g_{1}}^{2}+e_{n R_{1}}^{2}+\left(R_{1}+50\right)^{2} i_{\text {npreamp }}^{2}\right]\right. \\
& \left.+e_{n \text { reamp }}^{2}+e_{n R=1000}^{2}+1000^{2} i_{n \text { preamp }}^{2}\right\} \text {. }
\end{aligned}
$$

Considering an AM signal at the LIA input of the form $[1+A \cos (\omega t)] \cos \left(\omega_{0} t\right)$, where $\omega_{0}$ and $\omega$ are the carrier and modulating pulsation respectively, the filtered demodulated signal is $G_{L I A} A \cos (\omega t)$, where $G_{L I A}$ is related to the gain of the demodulation stage. This output signal is due to the sum of both upper and lower sidebands, each sideband being correlated with the same amplitude $\frac{A}{2}$. However, for an input white noise around the carrier, upper and lower sidebands are not correlated. Consequently, the output NSD is increased by a factor $G_{L I A} \sqrt{2}$, due to the quadratic sum of both the noise of the two sidebands. This effectively results in a decrease by a factor $\sqrt{2}$ of the signal to noise ratio ${ }^{1}$. Consequently, we express the equivalent output white noise power spectral density given at the sensor output by

$$
\begin{aligned}
e_{n_{X}}^{2}(\Delta f) & =\left(\frac{\sqrt{2} G_{L I A}}{G_{L I A} G}\right)^{2} e_{n L I A_{X}}^{2}\left(f_{0} \pm \Delta f\right) \\
& =\left(\frac{\sqrt{2}}{G}\right)^{2} e_{n L I A_{X}}^{2}\left(f_{0} \pm \Delta f\right)
\end{aligned}
$$

The next step is to evaluate the output sensitivity at the sensor output, represented by $T_{r_{X}}=\frac{\partial V_{\text {out }}(t)}{\partial B} \frac{1}{G_{L I A} G}$, in V/T. This is easily evaluated using $\frac{\partial Z_{i j}}{\partial B}$ and $Z_{i j_{0}}$ of Eq. 3, and from circuit elements. Equations 8a to 8d give the sensitivity of each configuration $(A, B, C$, or $D)$.

Finally, one can evaluate the equivalent magnetic noise spectral density of the setup, $b_{n_{X}}$, in $\mathrm{pT} / \sqrt{\mathrm{Hz}}$. It is defined as the ratio of the electronic noise spectral density (in $\mathrm{V} / \sqrt{\mathrm{Hz}}$ ) to the sensitivity (in V/T), $b_{n_{X}}=\frac{e_{n_{X}}}{T_{r_{X}}}$.

\section{PREliminary ReSUlts AND DisCUSSION}

With the help of the simplified model, developed in the previous section, we can now analyze the noise behavior for

\footnotetext{
${ }^{1}$ In case of demodulation with high carrier amplitude, signal to noise ratio may be reduced by the dynamics of the LIA.
}

the various excitation conditions, which is determined by the injection resistance, $R_{1}$, and the generator voltage amplitude, $e_{g_{1}}$. In this section, we consider a static working point, $B_{0}$, which sets static elements of the impedance matrix, $Z_{i j_{0}}$, to half maximum where differential variations of impedance, $\left.\frac{\partial Z_{i j}(B)}{\partial B}\right|_{B=B_{0}}$, are maximum. This is illustrated by red arrows on modulus curves of Fig. 2. Fig. 4 shows all noise contributions to the total NSD before demodulation, $e_{n L I A_{X}}$, for all four configurations as a function of $R_{1}$ and for an excitation $e_{g_{1}}=1 \mathrm{~V}_{\text {peak }}$. We may easily identify the dominant noise sources for each excitation condition. As expected, the NSD is dominated by generator noise for high excitation currents ( $R_{1}<1 \mathrm{k} \Omega$ for $e_{n_{A}}$ ) and by detection stage noise for lower excitation currents (combination of both preamplifier voltage noise, $e_{\text {npreamp }}$, and $1 \mathrm{k} \Omega$ resistor noise). Equivalent input voltage NSDs after demodulation, $e_{n_{X}}$, of each configuration, are summarized in Fig. 5.

Figure 6 shows the sensitivity versus excitation level for all four configurations. Note that the very high intrinsic sensitivity $\frac{\partial Z_{22}}{\partial B}$, shown in Fig. 2, does not necessarily lead to the highest output sensitivity for low values of $R_{1}$ because of the high value of $Z_{22_{0}}$. This, among other things, illustrates the necessity of fully understanding the entire system in order to choose the optimal conditioning for each configuration.

Finally, Fig. 7 shows the calculated equivalent magnetic noise levels in $\mathrm{pT} / \sqrt{\mathrm{Hz}}$ as a function of $R_{1}$. The figure shows non-trivial behavior of the noise with respect to electronic conditioning. It appears that the $B$ configuration (GMI $\rightarrow$ Coil) is the most promising for the set of parameters considered here. The optimal operating point (excitation) is located in the lowest flat part of the curve, where the dominating noise is due to the excitation stage. It is important to point out that, for an equivalent white NSD dominated by excitation noise, it is useless to further increase the excitation amplitude (in the linear regime) to produce higher response, because the noise level will increase as the sensitivity does. For the lowest values of $R_{1}$, the current flowing through the sample 


$$
\begin{aligned}
T_{r_{A}}\left(f_{0}\right) & \approx \frac{\partial\left|Z_{11}\left(f_{0}\right)\right|}{\partial B} \frac{e_{g_{1}}\left(R_{1}+50\right)}{\left(\left|Z_{11_{0}}\left(f_{0}\right)\right|+R_{1}+50\right)^{2}}, \\
T_{r_{B}}\left(f_{0}\right) & \approx \frac{e_{g_{1}}}{\left|Z_{11_{0}}\left(f_{0}\right)\right|+R_{1}+50}\left(\frac{\partial\left|Z_{21}\left(f_{0}\right)\right|}{\partial B}-\frac{\left|Z_{21_{0}}\left(f_{0}\right)\right|}{\left|Z_{11_{0}}\left(f_{0}\right)\right|+R_{1}+50} \frac{\partial\left|Z_{11}\left(f_{0}\right)\right|}{\partial B}\right) \\
& \approx \frac{e_{g_{1}}}{\left|Z_{11_{0}}\left(f_{0}\right)\right|+R_{1}+50} \frac{\partial\left|Z_{21}\left(f_{0}\right)\right|}{\partial B}, \\
T_{r_{C}}\left(f_{0}\right) & \approx \frac{e_{g_{1}}}{\left|Z_{22_{0}}\left(f_{0}\right)\right|+R_{1}+50}\left(\frac{\partial\left|Z_{12}\left(f_{0}\right)\right|}{\partial B}-\frac{\left|Z_{12_{0}}\left(f_{0}\right)\right|}{\left|Z_{22_{0}}\left(f_{0}\right)\right|+R_{1}+50} \frac{\partial\left|Z_{22}\left(f_{0}\right)\right|}{\partial B}\right) \\
& \approx \frac{e_{g_{1}}}{\left|Z_{22_{0}}\left(f_{0}\right)\right|+R_{1}+50} \frac{\partial\left|Z_{12}\left(f_{0}\right)\right|}{\partial B}, \\
T_{r_{D}}\left(f_{0}\right) & \approx \frac{\partial\left|Z_{22}\left(f_{0}\right)\right|}{\partial B} \frac{e_{g_{1}}\left(R_{1}+50\right)}{\left(\left|Z_{22_{0}}\left(f_{0}\right)\right|+R_{1}+50\right)^{2}} .
\end{aligned}
$$
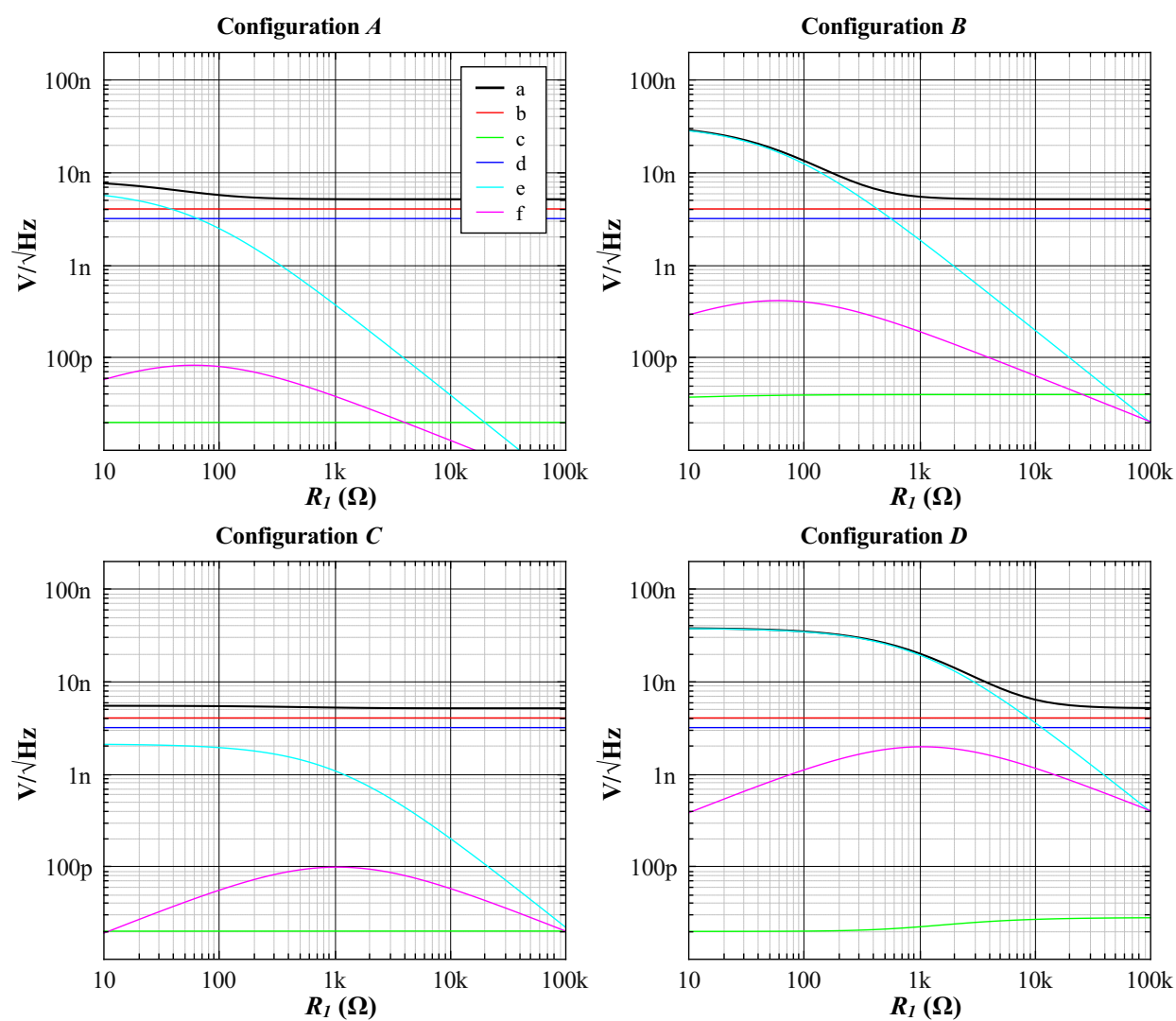

Figure 4. White noise spectral density, $\frac{e_{n L I A_{X}}}{G}$, at $\Delta f= \pm 10 \mathrm{kHz}$ from the carrier, $f_{0}$, expressed at the sensor output (a) for each configuration, $X$. Curves (b)-(f) indicate the various noise contributions to $\frac{e_{n L I A_{X}}}{G}$ as determined from Eqs. (6a) to (6d). These are the noise arising from the $1 \mathrm{k} \Omega$ resistor and network elements, (b), from the preamplifier current noise and network elements, (c), from the preamplifier voltage noise and network elements, (d), from generators and network elements, (e), and from the resistor $R_{1}$ and network elements, (f). These noise levels are shown versus the injection resistance $R_{1}$ for an excitation amplitude, $e_{g_{1}}$, of $1 \mathrm{~V}_{\text {peak }}$. 


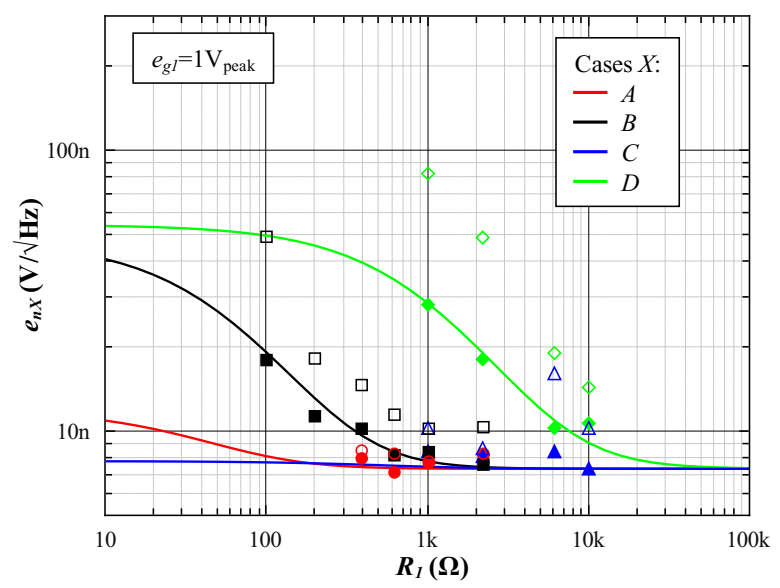

Figure 5. Theoretical (line) and measured (symbols) equivalent white noise spectral density, expressed at the sensor output, in $\mathrm{V} / \sqrt{\mathrm{Hz}}$ versus resistor value, $R_{1}$, at an excitation amplitude, $e_{g_{1}}$, of $1 \mathrm{~V}_{\text {peak }}$. Each curve corresponds to one of the four available configuration cases $(A, B, C$, or $D)$. Filled symbols are measured before LIA and considering an optimal demodulation as described in the present model whereas empty symbols are measured after SR844 LIA. In this later case, the noise spectral density could be limited by the dynamic of the used LIA. In all the following, we will consider experimental NSD measured as filled symbols.

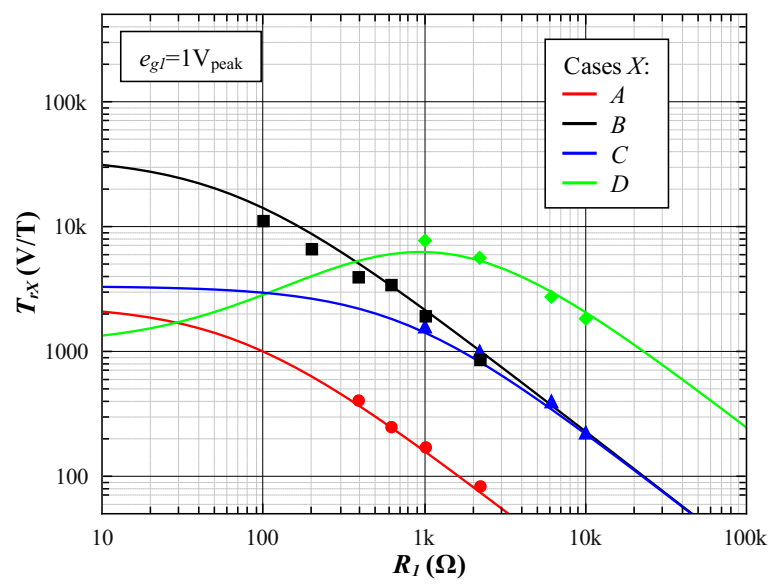

Figure 6. Theoretical (line) and measured (symbols) sensitivity after demodulation, expressed at the sensor output, in V/T versus resistor value, $R_{1}$, at an excitation amplitude, $e_{g_{1}}$, of $1 \mathrm{~V}_{\text {peak }}$. Each curve corresponds to one of the four available configuration $(A, B, C$, or $D)$.

becomes too large and leads to non-linear GMI behavior with some distortion. Nevertheless, previous work on non-linear GMI [20] suggests that the sensitivity will be maximum when sensing element is operated in the low non-linear range, just before the sensitivity starts to decrease. As a consequence, the best signal to noise ratio may be obtained at higher excitation current. However, this is not considered in the present model.

The model proposed here has been confronted with a series of noise measurements carried out on $\mathrm{CoFeSiB}$ microwire in the four configurations $A, B, C$, and $D$. The experimental details are given in Section II. The results are indicated by the symbols for each curve of Figs. 5 to 7 . The good agreement between measured data and the model suggests that the overall approach to noise modeling is sound and that the model includes the essential mechanisms limiting the performance of

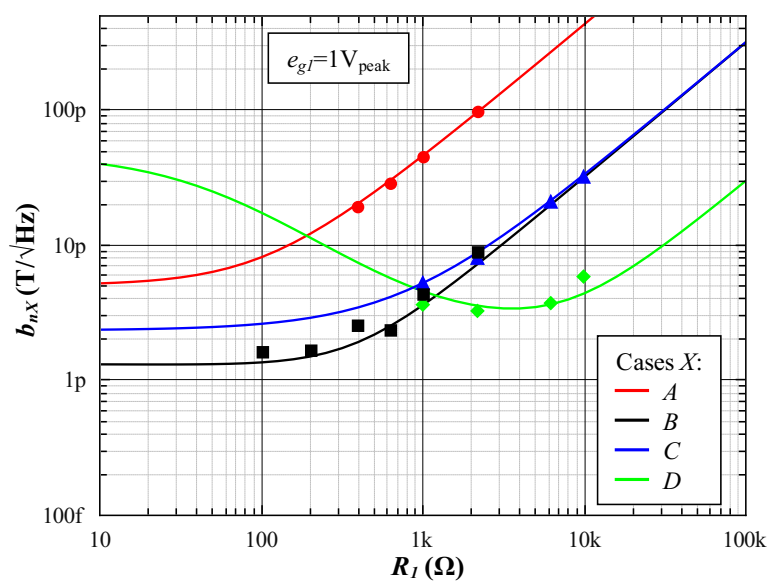

Figure 7. Theoretical (line) and measured (symbols) equivalent white magnetic noise spectral density at the sensor output, in $\mathrm{T} / \sqrt{\mathrm{Hz}}$ versus resistor value, $R_{1}$, at an excitation amplitude, $e_{g_{1}}$, of $1 \mathrm{~V}_{\text {peak }}$. Each curve corresponds to one of the four available configurations $(A, B, C$, or $D)$.

our GMI sensor. It also confirms, a posteriori, our assumption that the noise performance of the proposed design is still limited by electronic conditioning.

\section{NOISE IMPROVEMENT}

In the previous section, we considered a static working point, $B_{0}$, at which the $Z_{i j_{0}}$ are equal to their half maximum values. This choice is appropriate for validation of the model and discussion of the four configurations. Nevertheless, there is a small field range around this working point, $B_{0}$, in which the impedance variation is essentially linear. In other words, we may assume that the differential impedance variation, $\left.\frac{\partial Z_{i j}(B)}{\partial B}\right|_{B=B}$, remains constant in this range whereas $Z_{i j_{0}}$ varies with $B_{0}$. From Eqs. 6 a to $6 \mathrm{~d}$, we note that the values of $Z_{i j_{0}}$ affect the contribution of generators noise to the equivalent output NSD. These static values likewise affect the sensitivity as shown in Eqs. 8a to 8d.

Using the noise model developed here, we can evaluate the equivalent magnetic NSD at each limit of the linear range, defining a range of achievable values surrounding the curves of Fig 7. This is shown in Fig. 8.

Figure 8 clearly shows that improved noise performance may be obtained, in particular for the $B$ configuration (GMI $\rightarrow$ Coil) for which a decrease in $Z_{21_{0}}$ leads to a decrease in generator noise, the dominant noise source at high excitation current. This is confirmed by experimental measurements, as shown in Fig. 8, which compares results for the half maximum static working point (squares) with a static working point with a lower value of $Z_{21_{0}}$ (circles). As expected, there is a significant decrease of the equivalent magnetic NSD for the lowest values of excitation resistor $R_{1}$. The best measured value, around $1 \mathrm{pT} / \sqrt{\mathrm{Hz}}$, is not as good as expected. Indeed, for highest excitation current we cannot reach a value of $Z_{21_{0}}$ as low as expected without decreasing the sensitivity.

\section{CONCLUSION}

Following our earlier finding, that the GMI-coil configuration (Case $\mathrm{B}$ above) constitutes a promising approach to 


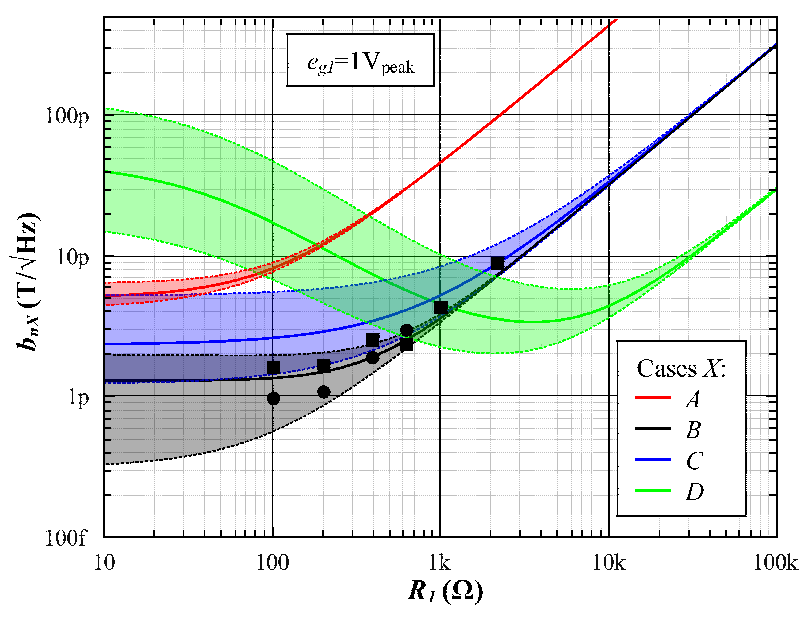

Figure 8. Theoretical (line and area) and measured (symbols) equivalent white magnetic noise spectral density expressed at the sensor output, in $\mathrm{T} / \sqrt{\mathrm{Hz}}$ versus resistor value, $R_{1}$, at an excitation amplitude, $e_{g_{1}}$, of $1 \mathrm{~V}_{\text {peak }}$. Each curve corresponds to one of the four available configurations $(A, B, C$, or $D)$. Colored areas illustrate achievable values for the static working point range in which the differential impedance variation is constant. Square and circle symbols indicate measured values in configuration $B$, for two different static working points, $B_{0}$. The squares correspond to the working point of Fig. 7, and the circles to a lower value of $Z_{21_{0}}$.

increasing the sensitivity of GMI effect sensors [14], we have demonstrated here that the noise performance may also be improved with this configuration. Indeed, our model allows us to understand the noise behavior with respect to four electronic conditioning configurations.

The good agreement between the model and experimental data validates our general approach. It thus allows us to extrapolate the results to other conditioning approaches and to predict the expected performance. For the present setup, the model leads to the determination of the most appropriate conditioning, providing the best possible performance for a given sensor configuration. It also highlights the dominant noise sources and limitations of GMI magnetometers. In the current state of the art, the noise performance of GMI magnetometers appears to be limited by that of the exciting generators. White noise levels as low as $1 \mathrm{pT} \sqrt{\mathrm{Hz}}$ have been obtained in this nonoptimal conditioning configuration, leaving room for further improvement.

\section{ACKNOWLEDGMENT}

The amorphous wire, based on CoFeSiB alloy, was kindly provided by H. Chiriac from National Institute of Research \& Development for Technical Physics, Romania.

\section{REFERENCES}

[1] M. Knobel, M. Vázquez, and L. Kraus, "Giant magnetoimpedance," Handbook of Magnetic Materials, vol. 15, pp. 1-92, 2003.

[2] K. Bushida, K. Mohri, and T. Uchiyama, "Sensitive and quick response micro magnetic sensor using amorphous wire MI element colpitts oscillator," IEEE Transactions on Magnetics, vol. 31, pp. 3134-3136, 1995.

[3] H. Hauser, L. Kraus, and P. Ripka, "Giant magnetoimpedance sensors," IEEE Instrumentation \& Measurement Magazine, vol. 4, pp. 28-32, 2001.
[4] K. Mohri, T. Uchiyama, L. Shen, C. Cai, L. Panina, Y. Honkura, and M. Yamamoto, "Amorphous wire and cmos ic-based sensitive micromagnetic sensors utilizing magnetoimpedance (mi) and stressimpedance (si) effects," IEEE Transactions on Magnetics, vol. 38, pp. 3063-3068, 2002.

[5] S. Yabukami, K. Kato, Y. Ohtomo, T. Ozawa, and K. Arai, "A thin film magnetic field sensor of sub-pt resolution and magnetocardiogram $(\mathrm{mcg})$ measurement at room temperature," Journal of Magnetism and Magnetic Materials, vol. 321, pp. 675-678, 2009.

[6] L. G. C. Melo, D. Ménard, A. Yelon, L. Ding, S. Saez, and C. Dolabdjian, "Optimization of the magnetic noise and sensitivity of giant magnetoimpedance sensors," Journal of Applied Physics, vol. 103, p. 033903, 2008.

[7] L. Ding, S. Saez, and C. Dolabdjian, "Low frequency giant magnetoimpedance magnetometer noise versus electronic conditioning," Sensor Letters, vol. 5, pp. 248-251, 2007.

[8] L. Ding, S. Nabily, S. Saez, J. Gieraltowski, and C. Dolabdjian, "Investigation of giant magnetoimpedance magnetic noise comparison," Sensor Letters, vol. 5, pp. 171-175, 2007.

[9] S. Sandacci, D. Makhnovskiy, L. Panina, K. Mohri, and Y. Honkura, "Off-diagonal impedance in amorphous wires and its application to linear magnetic sensors," IEEE Transactions on Magnetics, vol. 40, pp. 3505-3511, 2004.

[10] L. Kraus, "Off-diagonal magnetoimpedance in stress-annealed amorphous ribbons," Journal of Magnetism and Magnetic Materials, vol. 320, pp. e746-e749, 2008

[11] Z. Zhao, X. Li, J. Fan, H. Seet, X. Qian, and P. Ripka, "Comparative study of the sensing performance of orthogonal fluxgate sensors with different amorphous sensing elements," Sensors and Actuators A: Physical, vol. 136, pp. 90-94, 2007.

[12] I. Sasada, "Orthogonal fluxgate mechanism operated with dc biased excitation,” Journal of Applied Physics, vol. 91, pp. 7789-7791, 2002.

[13] L. Kraus, M. Malatek, and M. Dvorak, "Magnetic field sensor based on asymmetric inverse Wiedemann effect," Sensors and Actuators A: Physical, vol. 142, pp. 468-473, 2008.

[14] B. Dufay, S. Saez, C. Dolabdjian, D. Seddaoui, A. Yelon, and D. Ménard, "Improved GMI sensors using strongly-coupled thin pick-up coil," Sensors Letters, vol. 7, no. 3, pp. 334-338, 2009.

[15] L. Ding, S. Saez, C. Dolabdjian, L. G. C. Melo, A. Yelon, and D. Ménard, "Equivalent magnetic noise limit of low-cost GMI magnetometer," IEEE Sensors Journal, vol. 9, pp. 159-168, 2009.

[16] L. Ding, S. Saez, C. Dolabdjian, P. Ciureanu, L. G. C. Melo, A. Yelon, and D. Ménard, "Intrinsic giant magnetoimpedance noise reduction by dc bias," Sensor Letters, vol. 5, pp. 176-179, 2007.

[17] T. Lee and A. Hajimiri, "Oscillator phase noise: a tutorial," IEEE Journal of Solid-State Circuits, vol. 35, pp. 326-336, 2000.

[18] Function / Arbitrary waveform generator HP 33120A User's Guide, Hewlett-Packard Co., 1994.

[19] Low Noise Voltage Preamplifier EG\&G model 5113 data sheet, Signal Recovery Co.

[20] D. Seddaoui, D. Ménard, B. Movaghar, and A. Yelon, "Nonlinear electromagnetic response of ferromagnetic metals: Magnetoimpedance in microwires," Journal of Applied Physics, vol. 105, p. 083916, 2009. 